IJMMS 26:8 (2001) 485-496

PII. S016117120100240X

http://ijmms.hindawi.com

(c) Hindawi Publishing Corp.

\title{
THE GAME OF DOUBLE-SILVER ON INTERVALS
}

\author{
GERALD A. HEUER
}

(Received 29 June 2000)

\begin{abstract}
Silverman's game on intervals was analyzed in a special case by Evans, and later more extensively by Heuer and Leopold-Wildburger, who found that optimal strategies exist (and gave them) quite generally when the intervals have no endpoints in common. They exist in about half the parameter plane when the intervals have a left endpoint or a right endpoint, but not both, in common, and (as Evans had earlier found) exist only on a set of measure zero in this plane if the intervals are identical. The game of Double-Silver, where each player has its own threshold and penalty, is examined. There are several combinations of conditions on relative placement of the intervals, the thresholds and penalties under which optimal strategies exist and are found. The indications are that in the other cases no optimal strategies exist.
\end{abstract}

2000 Mathematics Subject Classification. 91A05.

1. Introduction. The game of Double-Silver is a generalization of Silverman's game, so we begin with a brief description of the latter and a brief survey of the literature on it. Silverman's game is a two-person zero-sum game in which, roughly speaking, the player with the higher number wins, unless it is too much higher than the other, in which case the high number loses. More precisely, players I and II have pure strategy sets $S_{\text {I }}$ and $S_{\text {II }}$ which may be somewhat arbitrary sets of positive real numbers. There are two parameters, the threshold, $T>1$, and the penalty, $v>0$. In a play of the game, player I chooses a number $x$ from $S_{\text {I }}$, player II chooses a number $y$ from $S_{\text {II }}$, and the higher number wins 1 , unless it is at least $T$ times as large as the other number, in which case it loses $v$. Thus, the payoff function $K_{s}(x, y)$ is given by

$$
K_{s}(x, y):= \begin{cases}v & \text { if } y \geq T x \\ -1 & \text { if } x<y<T x \\ 0 & \text { if } x=y \\ 1 & \text { if } y<x<T y \\ -v & \text { if } x \geq T y .\end{cases}
$$

A special case of this game with $S_{\mathrm{I}}=S_{\mathrm{II}}$ equal to the positive integers, with $v=$ 1 and special values of $T$, was introduced in the 1970s by David Silverman. Evans [1] analyzed the game for $S_{\mathrm{I}}=S_{\mathrm{II}}=(A, B)$, an open interval, finding that optimal mixed strategies exist only under very stringent conditions on the parameters, and obtaining optimal strategies when these conditions are fulfilled. Evans and Heuer [2] examined the symmetric game $\left(S_{\mathrm{I}}=S_{\mathrm{II}}\right)$ on discrete strategy sets (no accumulation points) and obtained explicit optimal mixed strategies except for some cases when 
the penalty $v$ is too near to zero. The asymmetric game on discrete sets is studied in $[3,5,6,8,9,11,12]$, and the game on intervals is studied in $[5,7,10,11]$. The monograph [11] is the best and most complete reference on the subject, superseding much of the earlier work.

In the game on intervals, it turns out that it is precisely the equality of the two intervals which makes it difficult for optimal mixed strategies to exist. The quadrant $\{(T, v): T>1, v>0\}$ roughly divides into two parts (each of unbounded measure), in one of which no optimal strategies exist when the left endpoints are equal, and in the other of which none exist when the right endpoints are equal. When both are equal (i.e., $S_{\mathrm{I}}=S_{\text {II }}$ ), optimal strategies exist only on the boundary between these two parts. When the intervals have no endpoints in common, optimal strategies nearly always exist. In all cases when they are known to exist, explicit optimal mixed strategies and game value are known (see [11, Chapters 2, 3, 4]).

In [4], a similar game on equal intervals is examined. Here the payoff is again a function of $y / x$, but instead of a step function, it is a continuous, piecewise linear function. The paper [13] reports on some experiments with a very simple case of Silverman's game, using university students as subjects.

In the game Double-Silver, each player has its own threshold and penalty. We use $T$ and $v$ for the threshold and penalty for player I; $U$ and $\mu$ for player II. Thus the payoff function is

$$
K(x, y):= \begin{cases}\mu & \text { if } y \geq U x, \\ -1 & \text { if } x<y<U x, \\ 0 & \text { if } x=y, \\ 1 & \text { if } y<x<T y, \\ -v & \text { if } x \geq T y .\end{cases}
$$

The thresholds $T$ and $U$ are any numbers larger than 1 , and the penalties $\mu$ and $\nu$ can be any positive numbers. Let

$$
u=\mu+1, \quad v=v+1 .
$$

We adopt the usual convention that player I has the interval with the minimum left endpoint, which, without loss of generality, we may take to be 1 . Thus the interval for player I will be $S_{\mathrm{I}}=(1, B)$ and that for player II, $S_{\mathrm{II}}=(A, D)$, with $1 \leq A$. We denote by $E(F, G)$ the expected payoff when player I uses strategy $F$ and player II uses $G$.

2. The case $U<A$. Note that if $U<A$, player I can force player II to "bust" by choosing from the interval $(1, A / U]$. We normally assume that $U>A$, but we first treat briefly the case $U<A$, which parallels [11, Theorem 3.32].

THEOREM 2.1. Suppose that

$$
1<U<A, \quad B \leq D .
$$

(i) If a game value $V$ exists, then $V=\mu$, and every strategy on $(1, A / U]$ is optimal for player $I$.

(ii) If $\mu \geq 1$, then $V=\mu$, and every strategy for player II is optimal.

(iii) If $D>B$, then $V=\mu$ and pure strategy $B$ is optimal for player II. 
Proof. If $1<x \leq A / U$, then for every $y$ in $S_{\text {II }}=(A, D)$ we have $y>A \geq U x$ and therefore $K(x, y)=\mu$. If $\mu \geq 1$ there are no payoffs larger than $\mu$, and (ii) clearly follows. To prove (i) we show that player I has no strategy $F$ for which $E(F, G)>\mu$ for every strategy $G$ on $S_{\text {II. }}$. To this end, let $F$ be any mixed strategy on $(1, B)$, and let $f_{1}, f_{2}$, and $f_{3}$ be the probabilities which $F$ assigns to $(1, A / U],(A / U, A]$, and $(A, B)$, respectively. If $f_{3}=0$, then $E(F, G) \leq \mu$ for every strategy $G$ on $S_{\text {II }}$ since $K(x, y) \leq \mu$ whenever $x<y$. Thus, suppose that $f_{3}>0$. Let $F_{0}$ be the mixed strategy on $(A, B)$ obtained by multiplying the density for the part of $F$ on $(A, B)$ by $1 / f_{3}$. Since it cannot be the case that $E\left(F_{0}, G\right)>0$ for every strategy $G$ on $(A, B)$, there is a strategy $G_{0}$ on $(A, B)$ such that $E\left(F_{0}, G_{0}\right) \leq 0$. (Indeed, $E\left(F_{0}, F_{0}\right)=0$.) For $x$ in $(A / U, A]$ and $y$ in $[A, B), K(x, y)$ is $\mu, 0$, or -1 , so $K(x, y) \leq \mu$. Thus

$$
E\left(F, G_{0}\right) \leq \mu f_{1}+\mu f_{2}+0 f_{3} \leq \mu
$$

and (i) follows. For (iii), note that for every $x$ in $(1, B), K(x, B) \leq \mu$. Since player I has a strategy $F$ with $E(F, y)=\mu$ for all $y$ in $(A, D)$, (iii) follows.

3. Games with $A>1$ in which penalty $v$ does not occur. In our next group of theorems, we shall have optimal strategies for player I all lying within the interval $(1, A T)$. This means that the penalty payoff $v$ does not occur, since with $y>A$ and $x<A T$ we always have $x<T y$. The following probability vectors $P_{n}$ and $Q_{n}$ will occur in describing optimal strategies.

$$
\begin{aligned}
P_{n} & =\left(p_{n, 1}, p_{n, 2}, \ldots, p_{n, n}\right) \\
& =\frac{1}{u^{n-1}+2 u^{n-2}+\cdots+2^{n-1}}\left(2^{n-1}, 2^{n-2} u, \ldots, 2 u^{n-2}, u^{n-1}\right),
\end{aligned}
$$

(recall from (1.3) that $u=\mu+1$ ), and

$$
Q_{n}=\left(q_{n, 1}, q_{n, 2}, \ldots, q_{n, n}\right)=\left(p_{n, n}, p_{n, n-1}, \ldots, p_{n, 1}\right)
$$

These are optimal strategies for a certain finite matrix game, as stated in the following lemma.

LEMMA 3.1. For the $n \times n$ game with payoff matrix

$$
H=\left(\begin{array}{cccccc}
-1 & \mu & \mu & \cdots & \mu & \mu \\
1 & -1 & \mu & \cdots & \mu & \mu \\
1 & 1 & -1 & \cdots & \mu & \mu \\
\vdots & \vdots & \vdots & \ddots & \vdots & \vdots \\
1 & 1 & 1 & \cdots & -1 & \mu \\
1 & 1 & 1 & \cdots & 1 & -1
\end{array}\right)
$$

the game value is

$$
V_{n}=\frac{u^{n-1}+2 u^{n-2}+\cdots+2^{n-2} u-2^{n-1}}{u^{n-1}+2 u^{n-2}+\cdots+2^{n-2} u+2^{n-1}}
$$


and optimal strategies for players I and II are given by the vectors $P_{n}$ and $Q_{n}$, respectively, of (3.1) and (3.2). In fact, the dot product of $P_{n}$ with each column of $H$ is equal to $V_{n}$, as is the dot product of $Q_{n}$ with each row of $H$.

Proof. It suffices to prove the last statement. The dot product of $P_{n}$ with the first column of $H$ is clearly equal to $V_{n}$. For $1 \leq r \leq n$, the difference $H_{\cdot_{r+1}}-H_{\cdot r}$ of the $(r+1)$ th and the $r$ th columns of $H$ is

$$
(0, \ldots, 0, u,-2,0, \ldots, 0)^{t},
$$

where $u=\mu+1$ is in the $r$ th position. The dot product

$$
P \cdot(H \cdot r+1-H \cdot r)=u p_{n, r}-2 p_{n, r+1}=0,
$$

so $P H_{. r}=V$ for each $r, 1 \leq r \leq n$. The corresponding fact for $Q$ is clear from the symmetry.

THEOREM 3.2. With $S_{\mathrm{I}}=(1, B), S_{\mathrm{II}}=(A, D)$, let $n$ be the (positive) integer such that

$$
U^{n-1}<T \leq U^{n}
$$

and assume that

$$
\begin{gathered}
1<A<U \leq U^{n}<\min \{B, A T\}, \\
D>A T, \\
u^{n} v \geq 2^{n+1} .
\end{gathered}
$$

Then the value of the game is $V_{n+1}$ (see (3.4)), and the following piecewise uniform mixed strategies $F$ and $G$ are optimal for players I and II, respectively:

$$
\begin{gathered}
F=P_{n+1} \quad \text { on }\left((1, \rho),(U, U \rho), \ldots,\left(U^{n}, U^{n} \rho\right)\right), \\
G=Q_{n+1} \quad \text { on }\left((A, A \rho),(A U, A U \rho), \ldots,\left(A U^{n-1}, A U^{n-1} \rho\right),(A T, A T \rho)\right),
\end{gathered}
$$

where $\rho>1$ but small enough that $\rho<A, A \rho<U, U^{n} \rho \leq \min \{B, A T\}$, and $A T \rho<D$.

The piecewise uniform strategies are to be understood as follows: the strategy $F$ distributes the probability $p_{n+1,1}$ uniformly over the interval $(1, \rho), p_{n+1,2}$ uniformly over interval $(U, U \rho)$, and so forth.

Note that (3.10) is satisfied, in particular, whenever $\mu \geq 1$ and $v \geq 1$.

Proof. Figure 3.1 depicts the interval layout and probability distributions for the case $n=3$. We first show that $E(F, y) \geq V_{n+1}$ for all $y>A$, where $E(F, y)$ denotes

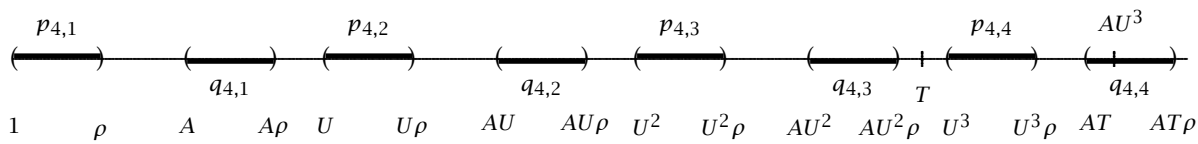

FigURE 3.1. The distributions $F$ and $G$ of Theorem 3.2, with $n=3$. 
the expected payoff of mixed strategy $F$ against pure strategy $y$. For $A<y \leq U$, $E(F, y)=-p_{n+1,1}+p_{n+1,2}+\cdots+p_{n+1, n+1}=V_{n+1}$. For $U^{k-1} \rho \leq y \leq U^{k}, 2 \leq k \leq n+1$,

$$
E(F, y)=\mu \sum_{j=1}^{k-1} p_{n+1, j}-p_{n+1, k}+\sum_{j=k+1}^{n+1} p_{n+1, j}=V_{n+1}
$$

by Lemma 3.1, and for $U^{k} \leq y \leq U^{k} \rho, 1 \leq k \leq n, E(F, y)$ is linear in $y$ and therefore constant at $V_{n+1}$, since that is the value at each endpoint. Thus $E(F, y)=V_{n+1}$ for $A<y \leq U^{n+1}$, and for $y>U^{n+1}, E(F, y)$ can only increase or remain constant. Thus $E(F, y) \geq V_{n+1}$ for all $y>A$.

Now we show that $E(x, G) \leq V_{n+1}$ for all $x>1$. For $1<x \leq A, E(x, G)=-q_{n+1,1}+$ $\mu \sum_{j=2}^{n+1} q_{n+1, j}=V_{n+1}$. For $A U^{k-1} \rho \leq x \leq A U^{k}, 1 \leq k \leq n-2$,

$$
E(x, G)=\sum_{j=1}^{k} q_{n+1, j}-q_{n+1, k+1}+\mu \sum_{j=k+2}^{n+1} q_{n+1, j}=V_{n+1}
$$

by Lemma 3.1. In particular,

$$
\begin{aligned}
E\left(A U^{n-2}, G\right) & =q_{n+1,1}+\cdots+q_{n+1, n-2}-q_{n+1, n-1}+\mu q_{n+1, n}+\mu q_{n+1, n+1}, \\
E\left(A U^{n-2} \rho, G\right) & =q_{n+1,1}+\cdots+q_{n+1, n-1}-q_{n+1, n}+\mu q_{n+1, n+1}=V_{n+1}, \\
E\left(A U^{n-1}, G\right) & \leq q_{n+1,1}+\cdots+q_{n+1, n-1}-q_{n+1, n}+\mu q_{n+1, n+1}=V_{n+1}
\end{aligned}
$$

(if $A T<A U^{n}$, then not all of $q_{n+1, n+1}$ is penalized). For $A U^{k} \leq x \leq A U^{k} \rho, 0 \leq k \leq$ $n-2, E(x, G)$ is linear in $x$ and therefore constant at $V_{n+1}$. For $A U^{n-1} \rho \leq x \leq A T$, $E(x, G)=q_{n+1,1}+\cdots+q_{n+1, n}-q_{n+1, n+1}=V_{n+1}$, and since again $E(x, G)$ is linear in $x$ for $A U^{n-1} \leq x \leq A U^{n-1} \rho$, it follows that $E(x, G) \leq V_{n+1}$ throughout this interval as well. At $x=A T \rho$, we have

$$
\begin{aligned}
E(A T \rho, G) & =-v q_{n+1,1}+q_{n+1,2}+\cdots+q_{n+1, n+1} \\
& =\frac{-v u^{n}+2 u^{n-1}+4 u^{n-2}+\cdots+2^{n-1} u+2^{n}}{u^{n}+2 u^{n-1}+4 u^{n-2}+\cdots+2^{n-1} u+2^{n}} \\
& =\frac{\left[2^{n+1}-(v+1) u^{n}\right]+\left(u^{n}+2 u^{n-1}+4 u^{n-2}+\cdots+2^{n-1} u-2^{n}\right)}{u^{n}+2 u^{n-1}+4 u^{n-2}+\cdots+2^{n-1} u+2^{n}} \\
& =\frac{2^{n+1}-u^{n} v}{u^{n}+2 u^{n-1}+4 u^{n-2}+\cdots+2^{n-1} u+2^{n}}+V_{n+1} \leq V_{n+1},
\end{aligned}
$$

since $u^{n} v \geq 2^{n+1}$ by hypothesis (3.10). For $x \leq A T \leq A T \rho, E(x, G)$ is linear in $x$ so it remains less than or equal to $V_{n+1}$. Finally, for $x>A U^{n} \rho, E(x, G)$ can only decrease or remain constant, so we have $E(x, G) \leq V_{n+1}$ for all $x>1$.

If we change condition (3.8) to make $B \leq U^{n}$ instead of $U^{n}<B$, and require $B<D$, we get a similar result, but using the probability vectors $P_{n}$ and $Q_{n}$ instead of $P_{n+1}$ and $Q_{n+1}$, and with game value $V_{n}$ in place of $V_{n+1}$. This result is given in the next theorem.

THEOREM 3.3. Let $S_{\text {I }}$ and $S_{\text {II }}$ be strategy sets and $n$ be defined as in (3.7). Assume that

$$
\begin{gathered}
1<A<\min \{T, U\}, \quad U^{n-1}<T<B \leq U^{n}, \\
B \leq A T, \quad B<D .
\end{gathered}
$$


Then the value of the game is $V_{n}$, and the following piecewise uniform mixed strategies $F$ and $G$ are optimal for players I and II, respectively:

$$
\begin{aligned}
& F=P_{n} \quad \text { on }\left((1, \rho),(U, U \rho), \ldots,\left(U^{n-2}, U^{n-2} \rho\right),\left(\frac{B}{\rho}, B\right)\right), \\
& G=Q_{n} \quad \text { on }\left(\left(\frac{U}{\rho}, U\right),\left(\frac{U^{2}}{\rho}, U^{2}\right), \ldots,\left(\frac{U^{n-1}}{\rho}, U^{n-1}\right),\{B\}\right), \text { if } B<U^{n},
\end{aligned}
$$

and if $B=U^{n}$, replace $\{B\}$ by $(B / \rho, B)$, where $\rho>1$ but small enough that $\rho<A<U / \rho$, $U^{n-1} \rho<T$, and if $B<U^{n}$ then $B<U^{n} / \rho$.

Proof. Figure 3.2 depicts the layout for $n=4$.

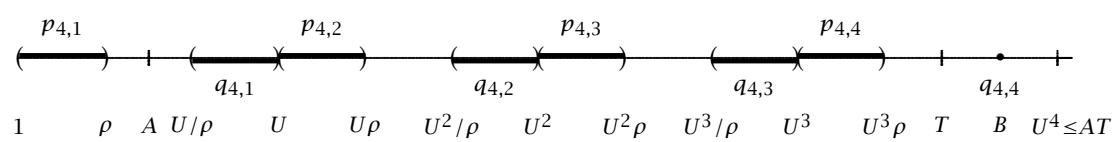

FIGURE 3.2. The distributions $F$ and $G$ of Theorem 3.3, with $n=4$.

We show first that $E(F, y) \geq V_{n}$ for all $y>A$. For $A<y \leq U$,

$$
E(F, y)=-p_{n, 1}+p_{n, 2}+\cdots+p_{n, n}=V_{n} .
$$

For $U^{k-1} \rho \leq y \leq U^{k}, 2 \leq k \leq n$,

$$
E(F, y)=\mu \sum_{j=1}^{k-1} p_{n, j}-p_{n, k}+\sum_{j=k+1}^{n} p_{n, j}=V_{n} .
$$

For $U^{k} \leq y \leq U^{k} \rho, 1 \leq k \leq n-1, E(F, y)$ is linear in $y$, and is equal to $V_{n}$ at both ends, so it has constant value $V_{n}$. For $y>U^{n-1} \rho, E(F, y)$ can only increase or remain constant. Thus $E(F, y) \geq V_{n}$ for all $y>A$.

Now we show that $E(x, G) \geq V_{n}$ for all $x$ in $(1, B)$. Assume first that $B=U^{n}$. For $U^{k-1} \leq x \leq U^{k} / \rho, 1 \leq k \leq n-1$,

$$
E(x, G)=\sum_{j=1}^{k-1} q_{n, j}-q_{n, k}+\mu \sum_{j=k+1}^{n} q_{n, j}=V_{n},
$$

and for $U^{n-1} \leq x<B$,

$$
E(x, G)=q_{n, 1}+\cdots+q_{n, n-1}-q_{n, n}=V_{n} .
$$

For $U^{k} / \rho \leq x \leq U^{k}, 1 \leq k \leq n-1, E(x, G)$ is linear in $x$, and is equal to $V_{n}$ at both ends, so it has constant value $V_{n}$. Thus we have $E(x, G)=V_{n}$ for all $x, 1<x<B$. If $B<U^{n}$, then in the case of $k=n-1$ in (3.20) instead of

$$
E(x, G)=\sum_{j=1}^{n-2} q_{n, j}-q_{n, n-1}+\mu q_{n, n},
$$

part of $q_{n, n}$ might be multiplied by $(-1)$ instead of $\mu$, making $E(x, G)<V_{n}$. Then as $x$ moves across $\left(U^{n-1} / \rho, U^{n-1}\right), E(x, G)$ is increasing linearly to the value $V_{n}$ at the right end. Again we have $E(x, G) \leq V_{n}$ for all $x$ with $1<x<B$. 
We may also drop condition (3.9) in Theorem 3.2 if we require $D>B$. We give the details in the next theorem.

THEOREM 3.4. Let $S_{\mathrm{I}}$ and $S_{\text {II }}$ be strategy sets and let $n$ be as defined in (3.7). Assume, as in Theorem 3.2, that

$$
1<A<U \leq U^{n}<\min \{B, A T\}, \quad u^{n} v \geq 2^{n+1},
$$

but in place of (3.9), that

$$
B<D \leq U^{n} A .
$$

Then the value of the game is $V_{n+1}$, and the following piecewise uniform mixed strategies $F$ and $G$ are optimal for players I and II, respectively:

$$
\begin{aligned}
& F=P_{n+1} \quad \text { on }\left((1, \rho),(U, U \rho), \ldots,\left(U^{n}, U^{n} \rho\right)\right), \\
& G=Q_{n+1} \quad \text { on }\left((A, A \rho),(A U, A U \rho), \ldots,\left(A U^{n-1}, A U^{n-1} \rho\right),\{B\}\right),
\end{aligned}
$$

where $\rho>1$ but small enough that $\rho<A, \rho<U, U^{n} \rho \leq \min \{B, A T\}$.

Proof. The proof of Theorem 3.2 applies with only minimal change. Strategy $F$ is unchanged, and that $E(F, y) \geq V_{n+1}$, for all $y>A$, did not depend on (3.9), so this remains true here. For strategy $G$, only the placement of the probability $q_{n+1, n+1}$ has changed. So long as this lies at or above $B$ and below $U^{n+1}, E(x, G)$ remains less than or equal to $V_{n+1}$ for all $x$ in $(1, B)$.

4. The case of equal left endpoints. The next theorem is the version of Theorem 3.3 with equal left endpoints, that is, $A=1$.

THEOREM 4.1. Let $S_{\text {I }}$ and $S_{\text {II }}$ be strategy sets, and assume that

$$
1=A<U \leq U^{n-1}<T \leq U^{n}, \quad B>U^{n-1},
$$

and either

$$
D \geq U^{n} \quad \text { or } \quad B<D<U^{n} .
$$

Let $P_{n}, Q_{n}$, and $V_{n}$ be as defined by (3.1), (3.2), and (3.4). Then the game value is $V_{n}$ and the following piecewise uniform mixed strategies $F$ and $G$ are optimal for players I and II, respectively:

$$
\begin{aligned}
& F=P_{n} \quad \text { on }\left((1, \rho),(U, U \rho), \ldots,\left(U^{n-1}, U^{n-1} \rho\right)\right), \\
& G=Q_{n} \quad \text { on }\left(\left(\frac{U}{\rho}, U\right),\left(\frac{U^{2}}{\rho}, U^{2}\right), \ldots,\left(\frac{U^{n}}{\rho}, U^{n}\right)\right), \text { if } D \geq U^{n} ;
\end{aligned}
$$

if $B<D<U^{n}$, replace the last interval in the $G$ distribution with $(D / \rho, D)$, where $\rho>1$ but is small enough that $1<\rho<\sqrt{U}, U^{n-1} \rho \leq T$, and if $B<D<U^{n}$, then $B<D / \rho$.

Proof. Figure 4.1 depicts the layout for $n=4$. We first show that $E(F, y) \geq V_{n}$ for all $y>1$. We use the expression $E(F, 1+)$ to denote $\lim _{y \rightarrow 1+} E(F, y)$. Starting 
from $E(F, 1+)=p_{n, 1}+\cdots+p_{n, n}>V_{n}, E(F, y)$ decreases linearly for $1<y<\rho$ to $E(F, \rho)=-p_{n, 1}+p_{n, 2}+\cdots+p_{n, n}=V_{n}$, and this value remains constant for $\rho \leq y \leq U$.

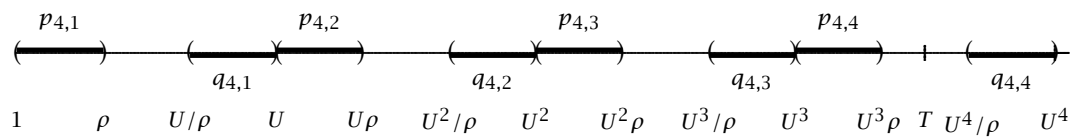

FigURE 4.1. The distributions $F$ and $G$ of Theorem 4.1 , with $n=4$.

For $U^{k-1} \rho \leq y \leq U^{k}$ with $2 \leq k \leq n, E(F, y)=\mu\left(p_{n, 1}+\cdots+p_{n, k-1}\right)-p_{n, k}+p_{n, k+1}+$ $\cdots+p_{n, n}=V_{n}$. For $U^{k} \leq y \leq U^{k} \rho$ with $1 \leq k \leq n-1, E(F, y)$ is linear in $y$, and therefore constant at $V_{n}$, since that is its value at both ends. For $y>U^{n}, E(F, y) \geq$ $E\left(F, U^{n}\right)=V_{n}$. Thus we have $E(F, y) \geq V_{n}$ for all $y>1$.

Now we show that $E(x, G) \leq V_{n}$ for all $x$ in $(1, B)$. For $1<x<U / \rho, E(x, G)=$ $-q_{n, 1}+\mu\left(q_{n, 2}+\cdots+q_{n, n}\right)=V_{n}$. For $U^{k-1} \leq x \leq U^{k} / \rho, 2 \leq k \leq n, E(x, G)=q_{n, 1}+$ $\cdots+q_{n, k-1}-q_{n, k}+\mu\left(q_{n, k+1}+\cdots+q_{n, n}\right)=V_{n}$. For $U^{k} / \rho \leq x \leq U^{k}, 1 \leq k \leq n, E(x, G)$ is linear in $x$ and is equal to $V_{n}$ at each end, so it has constant value $V_{n}$. The case $k=n$ here assumes that $D \geq U^{n}$. If $B<D<U^{n}$, then $B<U^{n} / \rho$, and the case $k=n$ does not occur. Thus $E(x, G) \leq V_{n}$ for all $x$ in $(1, B)$.

We recall from [1], or [11, Theorems 3.21, 3.24], that in Silverman's game on identical intervals $(A=1, B=D)$, optimal strategies exist when for some integer $n \geq 2, v$ is precisely equal to a certain value $v_{n}=\sec (\pi /(n+1))-1$ and $B>T^{n-1}$. With $n=2$ these conditions are $v=1$ and $B>T$. The optimal strategies and game value are unchanged if $B \neq D$ and both are larger than $T^{n-1}$ (cf. [11, Theorem 3.29]). There is a theorem similar to this one for the game of Double-Silver. Suppose that $U$ is a rational power of $T$; say, for some $a>1, U=a^{m}$, and $T=a^{n}$, where $m$ and $n$ are relatively prime positive integers, and that $\min \{B, D\} \geq a^{m+n}$. If $u^{n} v^{m}=(\mu+1)^{n}(v+1)^{m}=$ $2^{m+n}$, then optimal strategies exist for the game of Double-Silver on $(1, B) \times(1, D)$ and a simple algorithm for obtaining the optimal strategies may be given. We illustrate first with the special case where $m=3$ and $n=2$. Then $B$ and $D$ are each greater than or equal to $a^{5}$, and the optimal strategies are played on the interval $\left(1, a^{5}\right)$.

Let

$$
\begin{aligned}
P & =\left(p_{1}, p_{2}, p_{3}, p_{4}, p_{5}\right) \\
& =\frac{1}{16+8 v+4 v^{2}+2 u v^{2}+u v^{3}}\left(4 v^{2}, u v^{3}, 8 v, 2 u v^{2}, 16\right) \\
Q & =\left(q_{1}, q_{2}, q_{3}, q_{4}, q_{5}\right)=\left(p_{5}, p_{4}, p_{3}, p_{2}, p_{1}\right)
\end{aligned}
$$

Then the piecewise uniform mixed strategies $F$ and $G$ which assign $P$ and $Q$, respectively, to $\left((1, a)\left(a, a^{2}\right),\left(a^{2}, a^{3}\right),\left(a^{3}, a^{4}\right),\left(a^{4}, a^{5}\right)\right)$ are optimal, and the game value is

$$
V=\frac{16-8 v-4 v^{2}+2 u v^{2}-u v^{3}}{16+8 v+4 v^{2}+2 u v^{2}+u v^{3}}
$$


To see this, note first (bearing in mind that $U=a^{3}, T=a^{2}$ ) that

$$
\begin{gathered}
E(F, 1)=p_{1}+p_{2}-v p_{3}-v p_{4}-v p_{5}=U_{0} ; \\
E(F, a)=-p_{1}+p_{2}+p_{3}-v p_{4}-v p_{5}=U_{1} ; \\
E\left(F, a^{2}\right)=-p_{1}-p_{2}+p_{3}+p_{4}-v p_{5}=U_{2} ; \\
E\left(F, a^{3}\right)=-p_{1}-p_{2}-p_{3}+p_{4}+p_{5}=U_{3}=V ; \\
E\left(F, a^{4}\right)=\mu p_{1}-p_{2}-p_{3}-p_{4}+p_{5}=U_{4} ; \\
E\left(F, a^{5}\right)=\mu p_{1}+\mu p_{2}-p_{3}-p_{4}-p_{5}=U_{5} .
\end{gathered}
$$

Now $U_{0}-U_{1}=2 p_{1}-v p_{3}=0 ; U_{1}-U_{2}=2 p_{2}-v p_{4}=0 ; U_{2}-U_{3}=2 p_{3}-v p_{5}=0$; $U_{3}-U_{4}=-u p_{1}+2 p_{4}=0$; and $U_{4}-U_{5}=-u p_{2}+2 p_{5}=-u^{2} v^{3}+32$, which we have assumed to be 0 . Thus each $U_{k}=V, 0 \leq k \leq 5$. For $a^{k-1} \leq y \leq a^{k}, 1 \leq k \leq 5, E(F, y)$ is linear in $y$, and therefore has constant value $V$, since that is the value at each endpoint. Thus $E(F, y)=V$ for $1 \leq y \leq a^{5}$, and for $y>a^{5}, E(F, y) \geq E\left(F, a^{5}\right)$, so we have $E(F, y) \geq V$ for all $y \geq 1$.

For strategy $G$ we have

$$
\begin{gathered}
E(1, G)=-q_{1}-q_{2}-q_{3}+\mu q_{4}+\mu q_{5}=U_{5} ; \\
E(a, G)=q_{1}-q_{2}-q_{3}-q_{4}+\mu q_{5}=U_{4} ; \\
E\left(a^{2}, G\right)=q_{1}+q_{2}-q_{3}-q_{4}-q_{5}=U_{3} ; \\
E\left(a^{3}, G\right)=-v q_{1}+q_{2}+q_{3}-q_{4}-q_{5}=U_{2} ; \\
E\left(a^{4}, G\right)=-v q_{1}-v q_{2}+q_{3}+q_{4}-q_{5}=U_{1} ; \\
E\left(a^{5}, G\right)=-v q_{1}-v q_{2}-v q_{3}+q_{4}+q_{5}=U_{0} .
\end{gathered}
$$

Thus $E(x, G)=V$ at each of the interval endpoints, and by linearity, $E(x, G)=V$ for all $x$ with $1 \leq x \leq a^{5}$. For $x>a^{5}, E(x, G) \leq E\left(a^{5}, G\right)$, so $E(x, G) \leq V$ for all $x \geq 1$.

We note that $P$ and $Q$ in (4.4) are the optimal strategies for the matrix game with payoff matrix

$$
K=\left(\begin{array}{ccccc}
1 & -1 & -1 & -1 & \mu \\
1 & 1 & -1 & -1 & -1 \\
-v & 1 & 1 & -1 & -1 \\
-v & -v & 1 & 1 & -1 \\
-v & -v & -v & 1 & 1
\end{array}\right)
$$

We now give the general theorem.

THEOREM 4.2. Let $A=1$, and suppose that for some real number $a>1$ we have $U=a^{m}$ and $T=a^{n}$, where $m$ and $n$ are relatively prime positive integers. If

$$
\begin{gathered}
\min \{B, D\} \geq a^{m+n}, \\
u^{n} v^{m}=(\mu+1)^{n}(v+1)^{m}=2^{m+n},
\end{gathered}
$$

then optimal strategies and game value exist, and an algorithm may be given for calculating them. (The game value and optimal strategies are obtained in the proof.) 
Proof. Mixed strategies $F$ and $G$ will be defined as in the example preceding the theorem. Let

$$
\begin{gathered}
P=\left(p_{1}, p_{2}, \ldots, p_{m+n}\right), \\
Q=\left(q_{1}, q_{2}, \ldots, q_{m+n}\right)=\left(p_{m+n}, p_{m+n-1}, \ldots, p_{1}\right)
\end{gathered}
$$

be the probability vectors, with the idea that $F$ will assign the probability $p_{i}$ to be distributed uniformly over the interval $\left(a^{i-1}, a^{i}\right)$ for each $i$ with $1 \leq i \leq m+n$. Then

$$
\begin{aligned}
E(F, 1) & =p_{1}+\cdots+p_{n}-v\left(p_{n+1}+\cdots+p_{n+m}\right), \\
E(F, a) & =-p_{1}+p_{2}+\cdots+p_{n+1}-v\left(p_{n+2}+\cdots+p_{n+m}\right), \\
& \vdots \\
E\left(F, a^{m-1}\right) & =-p_{1}-\cdots-p_{m-1}+p_{m}+\cdots+p_{m+n-1}-v p_{m+n}, \\
E\left(F, a^{m}\right) & =-p_{1}-\cdots-p_{m}+p_{m+1}+\cdots+p_{m+n}, \\
E\left(F, a^{m+1}\right) & =\mu p_{1}-p_{2}-\cdots-p_{m+1}+p_{m+2}+\cdots+p_{m+n}, \\
E\left(F, a^{m+2}\right) & =\mu p_{1}+\mu p_{2}-p_{3}-\cdots-p_{m+2}+p_{m+3}+\cdots+p_{m+n}, \\
& \vdots \\
E\left(F, a^{m+n-1}\right) & =\mu\left(p_{1}+\cdots+p_{n-1}\right)-p_{n}-\cdots-p_{n+m-1}+p_{n+m}, \\
E\left(F, a^{m+n}\right) & =\mu\left(p_{1}+\cdots+p_{n}\right)-p_{n+1}-\cdots-p_{m+n} .
\end{aligned}
$$

Note that

$$
\begin{aligned}
E(F, 1)-E(F, a) & =2 p_{1}-v p_{n+1}, \\
E(F, a)-E\left(F, a^{2}\right) & =2 p_{2}-v p_{n+2}, \\
\vdots & \\
E\left(F, a^{m-1}\right)-E\left(F, a^{m}\right) & =2 p_{m}-v p_{n+m}, \\
E\left(F, a^{m}\right)-E\left(F, a^{m+1}\right) & =-u p_{1}+2 p_{m+1}, \\
\vdots & \\
E\left(F, a^{m+n-1}\right)-E\left(F, a^{m+n}\right) & =-u p_{n}+2 p_{m+n} .
\end{aligned}
$$

Now in order to choose $p_{1}, \ldots, p_{m+n}$ to make each of the differences in (4.13) equal to 0 , begin with $p_{m+n}=2^{m+n-1}$. Then to make $E\left(F, a^{m-1}\right)-E\left(F, a^{m}\right)=0$, put $p_{m}=$ $v p_{n+m} / 2=2^{m+n-2} v$; then (if $m>n$ ) $p_{m-n}=2^{m+n-3} v^{2}$, and so forth. As soon as subtracting $n$ from the subscript of $p$ results in a negative number, add $m+n$, and multiply by $u / 2$ instead of $v / 2$. Since $m$ and $n$ are relatively prime, we will use each equation of (4.13) except the last exactly once in order to determine each of $p_{1}$ through $p_{m+n-1}$ from our original choice of $p_{m+n}$. The last to be determined is $p_{n}=u^{n-1} v^{m}$, and then one condition (4.10) is exactly what is needed to get 0 in the last equation of (4.13). We then divide by the sum of the components of $P$ to make it a probability vector, and let $V$ be the common value of $E\left(F, a^{k}\right)$. For $a^{k-1} \leq y \leq a^{k}, 1 \leq k \leq n+m$, $E(F, y)$ is linear in $y$ and therefore $E(F, y)=V$ for $1 \leq y \leq a^{m+n}$. For $y>a^{m+n}$, $E(F, y) \geq E\left(F, a^{m+n}\right)$, so we have $E(F, y) \geq V$ for all $y \geq 1$. 
Now let $G$ be the mixed strategy for player II which distributes probability $q_{j}$ uniformly over the interval $\left(a^{j-1}, a^{j}\right), j=1, \ldots, m+n$. Then

$$
E(1, G)=-q_{1}-\cdots-q_{m}+\mu\left(q_{m+1}+\cdots+q_{m+n}\right)=E\left(F, a^{m+n}\right)=V,
$$

and in general,

$$
E\left(a^{k}, G\right)=E\left(F, a^{m+n-k}\right)=V, \quad 0 \leq k \leq m+n,
$$

so we have, reasoning as above, that $E(x, G) \leq V$ for all $x \geq 1$.

Thus strategies $F$ and $G$ are optimal and the game value is

$$
V=p_{1}+\cdots+p_{m-1}-p_{m}-\cdots-p_{m+n}
$$

and the theorem is proved.

5. Other cases. In all the above theorems but the last (Theorem 4.2), the first player has an optimal strategy in which the penalty $v$ never comes into play, and the game value is independent of $v$. (Note that $V_{n}$ defined in (3.4), depends on $u=\mu+1$ but not on $v=v+1$.)

The theorems above may well include all, or very nearly all, situations in which optimal strategies exist. These theorems, like most of the theorems about Silverman's game on intervals in $[10,11]$, were discovered using a technique for producing, from the game on a pair of intervals, a pair of (finite) matrix games, the solutions to which give highly useful information about the game on intervals. The values of these matrix games represent the best expected payoffs of which each of the two players can assure themselves when restricting to certain special types of mixed strategy distributions. Equality of the two values implies that optimal strategies exist and that the common value is the game value. Inequality does not, on the face of it, imply that no optimal strategies exist, but in the case of Silverman's game, we were nearly always able to use the information to prove nonexistence. This technique (for using certain matrix games to elicit information about games on intervals) seems likely to be more widely applicable, and we hope to examine this further in a future work. In every situation where we alter the hypotheses in such a way that none of the theorems above applies, we obtain different values for these two associated matrix games. This leads us to strongly suspect that no optimal strategies exist, but proofs would be rather long and technical and we have not actually carried out proofs in any of the cases.

\section{REFERENCES}

[1] R. J. Evans, Silverman's game on intervals, Amer. Math. Monthly 86 (1979), no. 4, 277-281. MR 81b:90156. Zbl 408.90091.

[2] R. J. Evans and G. A. Heuer, Silverman's game on discrete sets, Linear Algebra Appl. 166 (1992), 217-235. MR 93a:90082. Zbl 745.90080.

[3] G. A. Heuer, Odds versus evens in Silverman-type games, Internat. J. Game Theory 11 (1982), no. 3-4, 183-194. MR 85a:90233. Zbl 498.90089.

[4] - A family of games on $[1, \infty)^{2}$ with payoff a function of $y / x$, Naval Res. Logist. Quart. 31 (1984), no. 2, 229-249. MR 86b:90144. Zbl 535.90100.

[5] _ _ Reduction of Silverman-like games to games on bounded sets, Internat. J. Game Theory 18 (1989), no. 1, 31-36. MR 90c:90244. Zbl 674.90102. 
[6] G. A. Heuer and U. Leopold-Wildburger, Balanced Silverman Games on General Discrete Sets, Lecture Notes in Economics and Mathematical Systems, vol. 365, SpringerVerlag, Berlin, 1991. MR 94c:90002. Zbl 743.90117.

[7] __ Asymmetric Silverman games on intervals, working paper no. 27, Department of Business, University of Graz, 1992.

[8] _ Nonsingularity of some classes of matrices, and minimal solutions of Silverman's game on discrete sets, Math. Comput. Modelling 18 (1993), no. 12, 1-31. MR 94k:90152. Zbl 799.90149.

[9] __ Fibonacci-type sequences and minimal solutions of discrete Silverman games, Fibonacci Quart. 32 (1994), no. 1, 22-43. MR 95c:90126. Zbl 798.90135.

[10] - The advantage of deeper pockets in Silverman's game on intervals, Naval Res. Logist. 42 (1995), no. 1, 123-140. MR 95i:90100. Zbl 833.90128.

[11] _ Silverman's Game. A Special Class of Two-Person Zero-Sum Games, Lecture Notes in Economics and Mathematical Systems, vol. 424, Springer-Verlag, Berlin, 1995. MR 96f:90002. Zbl 854.90142.

[12] G. A. Heuer and W. D. Rieder, Silverman games on disjoint discrete sets, SIAM J. Discrete Math. 1 (1988), no. 4, 485-525. MR 90a:90224. Zbl 661.90101.

[13] O. Mueller, U. Leopold-Wildburger, and G. A. Heuer, Some experiments with Silverman's game, Cent. Eur. J. Oper. Res. Econ. 4 (1996), no. 1, 57-68. Zbl 959.91506.

Gerald A. Heuer: Department of Mathematics ANd Computer SCience, ConCordia ColLEGE, MOORHEAD, MN 56562, USA

E-mail address: heuer@cord.edu 


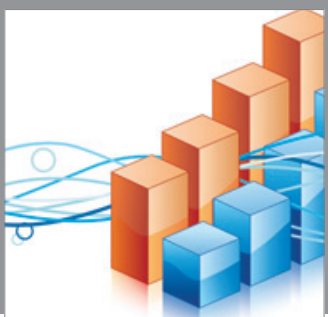

Advances in

Operations Research

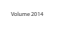

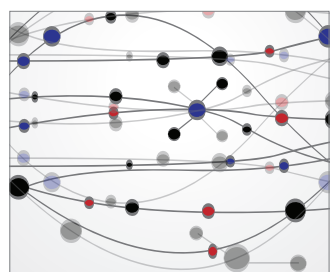

\section{The Scientific} World Journal
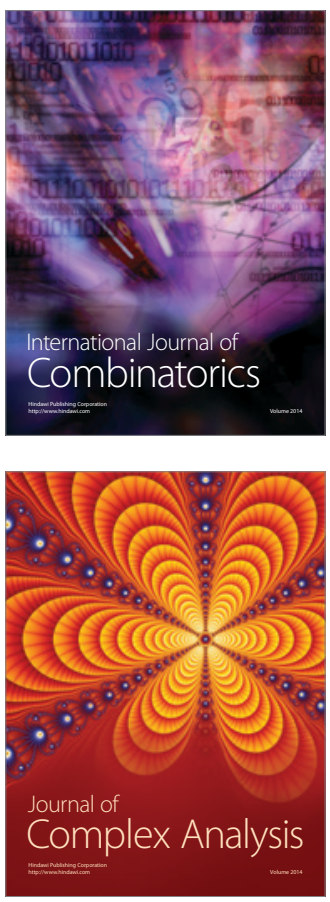

International Journal of

Mathematics and

Mathematical

Sciences
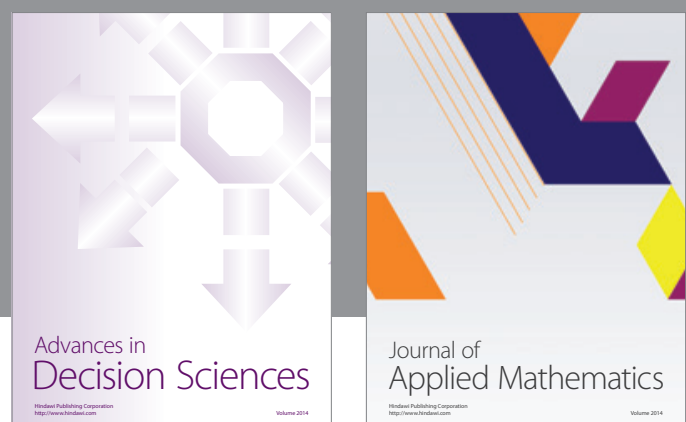

Journal of

Applied Mathematics
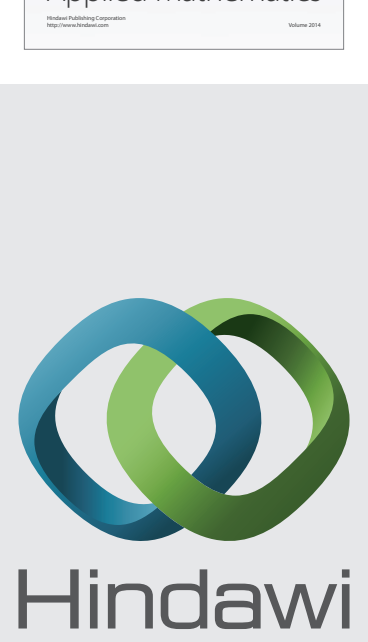

Submit your manuscripts at http://www.hindawi.com
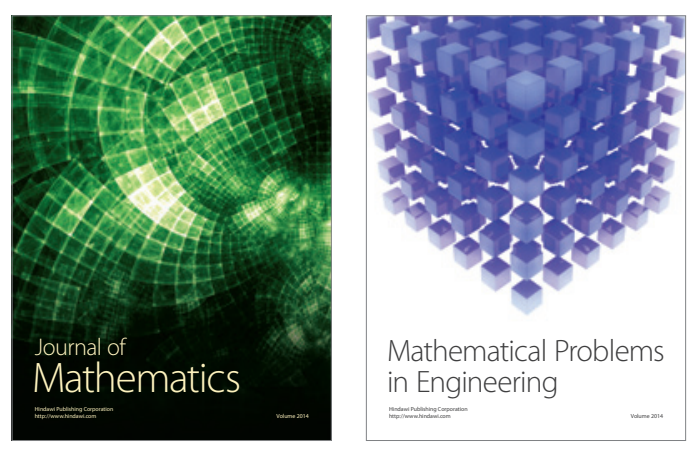

Mathematical Problems in Engineering
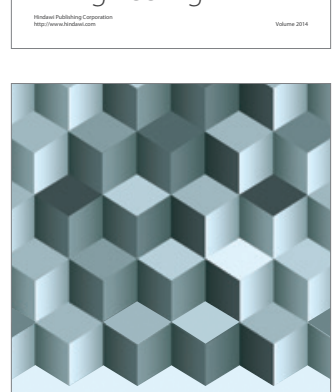

Journal of

Function Spaces
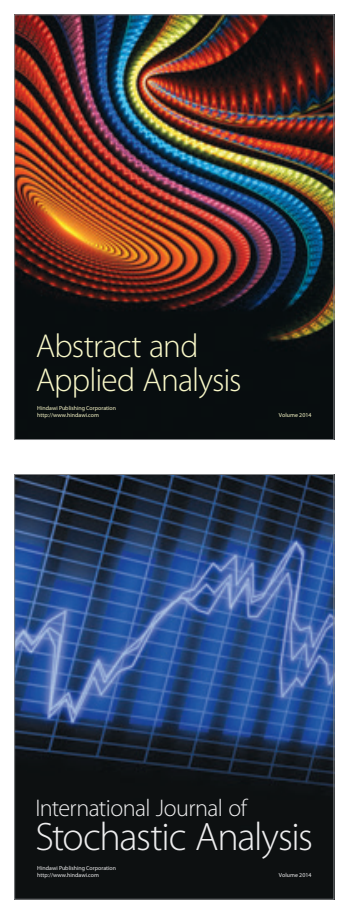

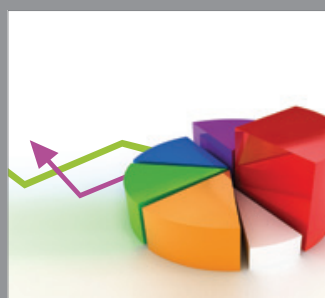

ournal of

Probability and Statistics

Promensencen
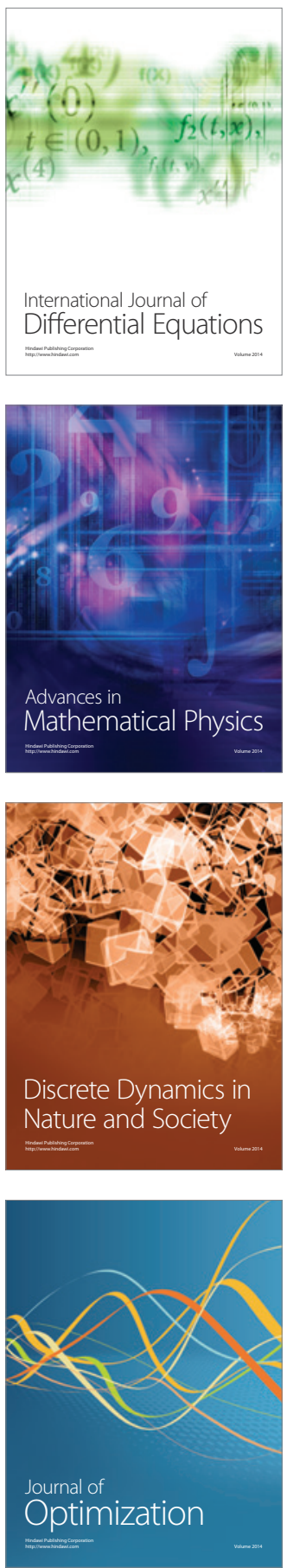